\title{
Involvement of Rho-associated protein
} kinase (ROCK) and bone morphogenetic protein-binding endothelial cell precursor-derived regulator (BMPER) in high glucose-increased alkaline phosphatase expression and activity in human coronary artery smooth muscle cells

Yuya Terao ${ }^{1}$, Seimi Satomi-Kobayashi ${ }^{1}$, Ken-ichi Hirata ${ }^{1}$ and Yoshiyuki Rikitake ${ }^{1,2^{*}}$

\begin{abstract}
Background: Vascular calcification is an independent risk factor for cardiovascular disease. Diabetes mellitus increases the incidence of vascular calcification; however, detailed molecular mechanisms of vascular calcification in diabetes mellitus remain unknown. We recently reported that bone morphogenetic protein-binding endothelial cell precursor-derived regulator (BMPER) regulates osteoblast-like trans-differentiation of human coronary artery smooth muscle cells (HCASMCs).

Methods: We investigated the effect of a hydroxymethylglutaryl-coenzyme A reductase inhibitor (statin), commonly used in patients with atherosclerotic diseases and diabetes mellitus, on alkaline phosphatase (ALP) mRNA expression in aortas of streptozotocin-induced diabetic mice. We also investigated the effects of the statin, Rho-associated protein kinase (ROCK) inhibitors and BMPER knockdown on ALP mRNA expression and activity in HCASMCs cultured in high glucose-containing media.

Results: Alkaline phosphatase mRNA expression was increased in aortas of streptozotocin-induced diabetic mice, and the increase was inhibited by rosuvastatin. ALP mRNA expression and activity were increased in HCASMCs cultured in high glucose-containing media, and the increases were suppressed by rosuvastatin. This suppression was reversed by the addition of mevalonate or geranylgeranyl pyrophosphate, but not farnesyl pyrophosphate. High glucose-increased ALP mRNA expression and activity were suppressed by ROCK inhibitors. Moreover, BMPER mRNA expression was increased in diabetic mouse aortas and in HCASMCs cultured in high glucose-containing media, but was not inhibited by rosuvastatin or ROCK inhibitors. Knockdown of BMPER suppressed high glucose-increased ALP activity, but not ROCK activity in HCASMCs.
\end{abstract}

Conclusions: There are at least two independent pathways in high glucose-induced ALP activation in HCASMCs: the Rho-ROCK signaling pathway and the BMPER-dependent pathway.

\footnotetext{
*Correspondence: rikitake@med.kobe-u.ac.jp

2 Division of Signal Transduction, Department of Biochemistry

and Molecular Biology, Kobe University Graduate School of Medicine,

7-5-1 Kusunoki-cho, Chuo-ku, Kobe 650-0017, Japan

Full list of author information is available at the end of the article
} 
Keywords: ALP, BMPER, High glucose, ROCK, Statin, Vascular smooth muscle cells

\section{Background}

Vascular calcification is one of the common pathological changes of atherosclerosis and is an independent risk factor for cardiovascular disease. The incidence of vascular calcification increases with aging, smoking, hemodialysis, and diabetes mellitus. However, detailed molecular mechanisms of vascular calcification in diabetes mellitus remain poorly understood. In diabetes mellitus, high glucose promotes trans-differentiation of vascular smooth muscle cells, which is thought to cause vascular calcification, and hyperinsulinemia and elevated advanced glycosylation end products may play a role in vascular calcification [1].

Hydroxymethylglutaryl-coenzyme A (HMG-CoA) reductase inhibitors, statins, are drugs that lower cholesterol levels by inhibiting HMG-CoA reductase. Statins are commonly used in patients with atherosclerotic diseases and diabetes mellitus. It is widely recognized that statins have pleiotropic effects unrelated to cholesterollowering effects, such as anti-inflammatory and antioxidative effects [2]. In addition to inhibiting cholesterol synthesis, statins also block the synthesis of isoprenoid intermediates such as farnesyl pyrophosphate (FPP) and geranylgeranyl pyrophosphate (GGPP) [3]. FPP and GGPP serve as important lipid attachments for the posttranslational modification of a variety of proteins, including small GTPases [4]. Modification with FPP is necessary for proper localization of Ras family proteins, whereas GGPP is required for Rho, Rab, and Rap family proteins. In vitro studies have demonstrated that statins inhibit Rho by inhibiting GGPP, and thereby suppress its effector Rho-associated protein kinase (ROCK) [5].

It was reported that statins inhibit in vitro calcification of vascular smooth muscle cells induced by inorganic phosphate [6], inflammatory mediators [7], vitamin $\mathrm{D}_{3}$ and warfarin [8], and transforming growth factor (TGF)- $\beta$ [9]. However, with regard to the inhibitory effects of statins on vascular calcification, conflicting results have been reported between in vitro research and clinical studies. Several clinical studies using electron beam tomography have shown that statins reduce coronary artery calcification [10-12]. However, three subsequent randomized trials using electron beam tomography have shown no inhibitory effects of statins on coronary artery calcification [13-15]. Furthermore, a recent analysis of eight randomized trials using intravascular ultrasonography showed that statins promoted coronary artery calcification [16].
Bone morphogenetic protein (BMP)-binding endothelial cell precursor-derived regulator (BMPER) is a secretory protein that is known to bind to BMP-2, 4 and 6 [17]. BMPER can work as either an activator or an inhibitor of BMP signaling, depending on its concentrations and environment [18]. We have reported that BMPER is a regulator of the osteoblast-like trans-differentiation of human coronary artery smooth muscle cells (HCASMCs) [19]. Knockdown of BMPER inhibits, whereas addition of BMPER enhances the osteoblast-like trans-differentiation of HCASMCs.

Here, we investigated the effects of a statin, ROCK inhibitors and BMPER knockdown on alkaline phosphatase (ALP) mRNA expression and activity in HCASMCs cultured in high glucose-containing media to examine the potentially critical roles of the Rho-ROCK signaling pathway and BMPER in vascular calcification in diabetes mellitus.

\section{Methods}

Mice

All animal experiments were approved by the Institutional Animal Care and Use Committee and carried out according to the Kobe University Animal Experimental Regulations (P140605). Streptozotocin (STZ)induced type I diabetic mice were produced as described previously [20] with slight modification. Briefly, male C57BL/6J mice (10-week-old) were intraperitoneally injected with STZ in distilled water $(200 \mathrm{mg} / \mathrm{kg}$; Wako, Osaka, Japan) or the equal volume of vehicle as a control. Blood samples were taken from mouse lateral tail vein and blood glucose was measured by OneTouch Ultra glucometer (LifeScan, Wayne, PA, USA). Fasting blood glucose was measured at 4 and 8 days after the first STZ injection, and additional STZ injections $(250 \mathrm{mg} / \mathrm{kg}$ ) were given when the fasting blood glucose was less than $250 \mathrm{mg} / \mathrm{dl}$. Rosuvastatin, kindly provided by AstraZeneca, was dissolved in drinking water, which was available ad libitum, and administered with $7.2 \mathrm{mg} /$ $\mathrm{kg}$ body weight/day for 14 days. After being anesthetized intraperitoneally with $2.5 \%$ 2,2,2-tribromoethanol $(1.6 \mathrm{ml} / 100 \mathrm{~g})$, the mice were transcardially perfused with physiological saline solution and the aortas were isolated.

\section{Cell culture}

Human coronary artery smooth muscle cells (Lonza, Basel, Switzerland) were cultured at $37{ }^{\circ} \mathrm{C}$ in Dulbecco's modified Eagle's medium (Nacalai, Kyoto, Japan) 
(glucose; $5.5 \mathrm{mM}$ ) supplemented with $15 \%$ fetal bovine serum (Life Technologies, Carlsbad, CA, USA), 100 IU/ $\mathrm{ml}$ penicillin and $100 \mathrm{~g} / \mathrm{ml}$ streptomycin (Nacalai) (normal glucose-containing media). High glucose-containing media (final $25 \mathrm{mM}$ ) was made by addition of $19.5 \mathrm{mM}$ glucose to normal glucose-containing media. In like manner, $19.5 \mathrm{mM}$ mannitol (Sigma-Aldrich, St. Louis, MO, USA) was added to normal glucose-containing media as an osmolality control (mannitol-containing media). Cells between passages 8 and 14 were used for the experiments. Unless otherwise noted, cells were cultured for the indicated time periods without changing media. In some experiments, HCASMCs were cultured with rosuvastatin $(10 \mu \mathrm{M})$, mevalonate (Sigma-Aldrich) $(100 \mu \mathrm{M})$, FPP (Sigma-Aldrich) $(10 \mu \mathrm{M})$, GGPP (SigmaAldrich) $(10 \mu \mathrm{M})$, fasudil (Wako) $(10 \mu \mathrm{M})$ and Y-27632 (Wako) $(10 \mu \mathrm{M})$. Human umbilical vein endothelial cells (HUVECs) (Lonza) were cultured at $37^{\circ} \mathrm{C}$ in the EGM-2 BulletKit (Lonza).

\section{Small interfering RNA (siRNA) experiments}

Knockdown of BMPER by siRNAs was performed as described previously [19]. HCASMCs were transfected with Stealth siRNAs for BMPER (Life Technologies) using Lipofectamine RNAiMAX (Life Technologies) according to the manufacturer's instructions. A Stealth siRNA non-silencing negative control (Life Technologies) was used as a control. Forty-eight hours after transfection, HCASMCs were subjected to each experiment.

\section{Real-time polymerase chain reaction (PCR)}

Real-time PCR was performed as described previously [19]. Total mRNAs were extracted from mouse aortas and HCASMCs using TRIzol Reagent (Life Technologies) and subjected to real-time PCR using a 7500 Real-Time PCR System (Life Technologies) with a SYBR Premix Ex Taq II (Tli RNaseH Plus) (Takara Bio, Otsu, Japan). The following primers were used: mouse ALP: forward, 5-ACACCTTGACTGTGGTTACTGCTG A-3 and reverse, 5-CCTTGTAGCCAGGCCCGTTA-3; mouse BMPER: forward, 5-ATTACCTGCTGCGTCTT GCT-3 and reverse, 5-TTCTCTCACGCACTGTGTCC-3; mouse GAPDH: forward, 5-GACCCCTTCATTGACCTC AACTAC-3 and reverse, 5-TTTCTTACTCCTTGGAGGC CATGT-3; and human ALP: forward, 5-GGACCAT TCCCACGTCTTCAC-3 and reverse, 5-CCTTGTAGCC AGGCCCATTG-3; human BMPER: forward, 5-AGG ACAGTGCTGCCCCAAATG-3 and reverse, 5-TACTGAC ACGTCCCCTGAAAG-3; human glyceraldehyde3-phosphate dehydrogenase (GAPDH): forward, 5-CT GATGCCCCCATGTTCGTC-3 and reverse, 5-CACCCTGT TGCTGTAGCCAAATTC-3. Primer pairs were purchased from Takara Bio. GAPDH was used for standardization, and the comparative threshold method was used to assess the relative abundance of the targets.

\section{ALP staining}

Alkaline phosphatase staining was performed essentially as described previously [19]. HCASMCs cultured in 24-well plates were washed with phosphate-buffered saline, and fixed in $4 \%$ paraformaldehyde for $2 \mathrm{~min}$. The cells were incubated in substrate working solution $[100 \mathrm{mg} / \mathrm{ml}$ naphthol AS-MX phosphatase (SigmaAldrich), $600 \mathrm{mg} / \mathrm{ml}$ fast red TR salt (Sigma-Aldrich), $0.5 \%$ (v/v) N,N-dimethylformamide, $2 \mathrm{mM} \mathrm{MgCl}_{2}$ and $0.1 \mathrm{M}$ Tris $-\mathrm{HCl} \mathrm{pH} \mathrm{8.8]} \mathrm{at} 37{ }^{\circ} \mathrm{C}$ for $20 \mathrm{~min}$. The cells were washed until the intense red color became indicative. The ratio of ALP-positive area was calculated from 10 wells, with at least 500 cells counted per well, using ImageJ software.

\section{Western blotting}

Western blotting was performed as described previously [21]. ROCK activity was measured by myosin phosphatase target subunit 1 (MYPT1) phosphorylation as described previously [22]. Rabbit anti-phospho-MYPT1 polyclonal antibody (\#4563, Cell Signaling Technology, Danvers, MA, USA), rabbit anti-MYPT1 polyclonal antibody (\#2634, Cell Signaling Technology) and anti-rabbit immunoglobulin G, horseradish peroxidase-linked whole antibody donkey (GE Healthcare Bioscience, Pittsburgh, PA, USA) were used at 1:1,000. The signals were detected using the Amersham Imager 600 (GE Healthcare Bioscience, Little Chalfont, UK). Densitometric analysis was performed using ImageJ software.

\section{Statistical analysis}

All experiments were performed at least three times, and the results are expressed as mean \pm standard error of the mean (SEM). Student's $t$-test was used when comparing differences between two groups. Differences between more than three groups were analyzed by one-way analysis of variance, followed by Tukey's or Dunnett's multiple comparison tests, as appropriate. Values of $P<0.05$ were considered significant.

\section{Results}

Rosuvastatin decreased ALP mRNA expression in aortas of STZ-induced type I diabetic mice

We investigated the effect of rosuvastatin on ALP expression in aortas of STZ-induced type I diabetic mice. Compared with control mice, ALP mRNA expression in aortas of STZ-induced type I diabetic mice given rosuvastatin was significantly increased $(\sim 2.0$-fold; $P<0.01)$. Administration of rosuvastatin significantly inhibited the increase in ALP mRNA expression $(P<0.05)$ (Fig. 1a). 


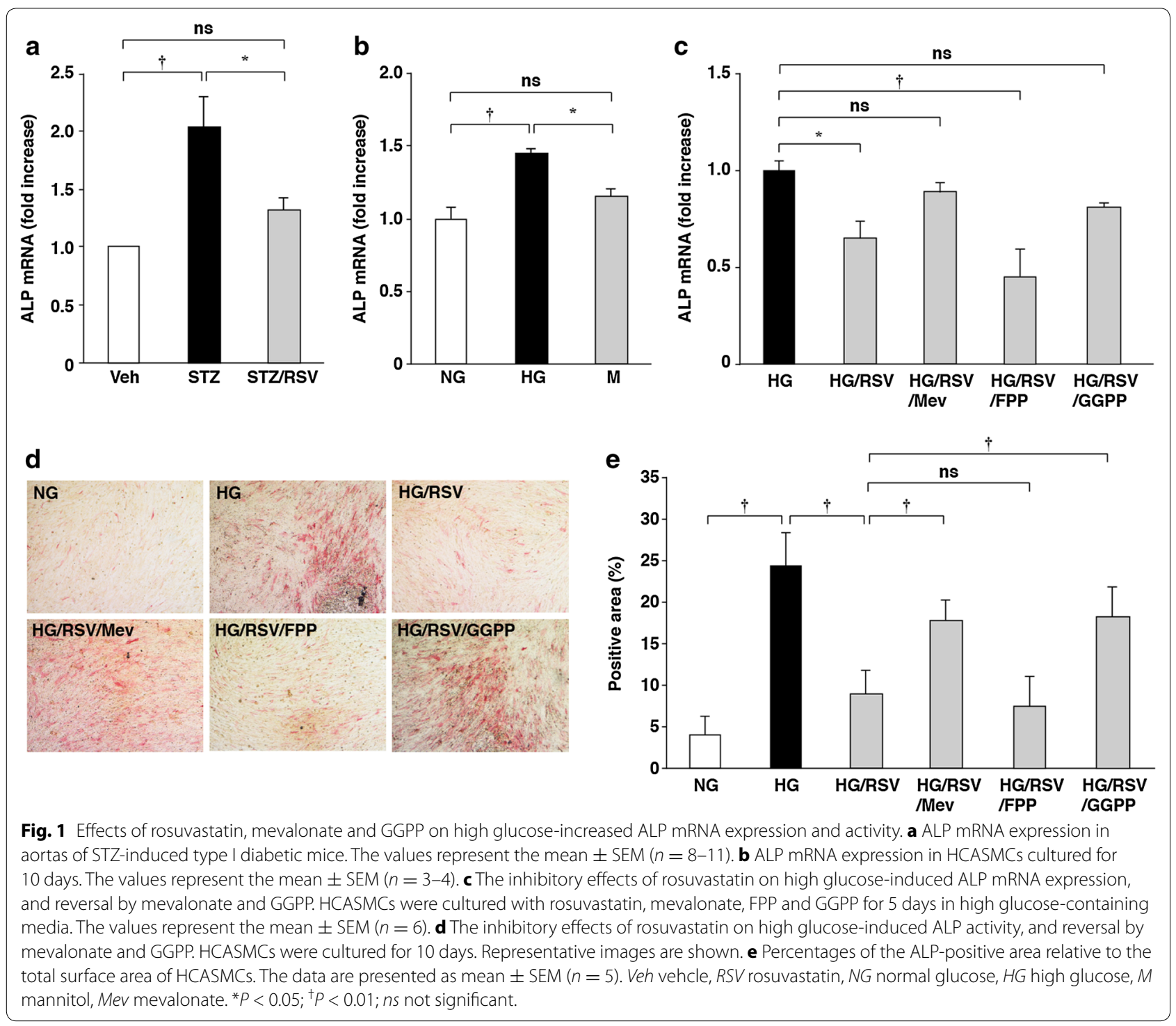

\section{Rosuvastatin suppressed high glucose-increased ALP mRNA expression and activity in HCASMCs}

To elucidate molecular mechanisms of the inhibition of ALP mRNA expression in diabetic mice by rosuvastatin, we performed in vitro experiments using cultured HCASMCs. When HCASMCs were cultured in high glucose-containing media, ALP mRNA expression and activity were significantly increased and these increases were significantly suppressed by rosuvastatin (Fig. 1b, d). Because statins show pleiotropic effects by inhibiting isoprenoid synthesis, the effects of mevalonate, FPP and GGPP on the rosuvastatin-suppressed ALP expression were examined. The suppression of ALP expression by rosuvastatin was reversed by the addition of mevalonate and GGPP, but not by the addition of FPP (Fig. 1c-e).
These results indicate that rosuvastatin suppresses high glucose-increased ALP mRNA expression and activity in HCASMCs, and that the effects of rosuvastatin are likely due to the inhibition of GGPP synthesis.

\section{ROCK inhibitors suppressed high glucose-increased ALP mRNA expression and activity in HCASMCs}

GGPP is required for geranylgeranylation of small G proteins such as Rho, Rac and Cdc42 [4]. In particular, inhibition of Rho and its downstream target, ROCK, has emerged as the principle mechanism underlying the pleiotropic effects of statins [22, 23]. We therefore focused on the role of the Rho-ROCK signaling pathway. To reveal whether ROCK is involved in high glucose-increased ALP expression and activity, the effects of specific ROCK 
inhibitors, fasudil and Y-27632, were examined. The increases in ALP mRNA expression and activity by cultivation in high glucose-containing media were effectively suppressed by the ROCK inhibitors fasudil and Y-27632 (Fig. 2a-c).

\section{BMPER was involved in high glucose-increased ALP activity in HCASMCs}

We then examined whether BMPER was involved in high glucose-increased ALP activity. BMPER mRNA levels were increased by cultivation in high glucose-containing media, but not by mannitol (Fig. 3a). The high glucose-increased ALP activity was suppressed by knockdown of BMPER by siRNAs (Fig. 3b, c). Furthermore, BMPER mRNA levels were increased in aortas of STZ-induced type I diabetic mice by 2.5 -fold $(P<0.05)$ (Fig. 4a). Collectively, these results show that BMPER mRNA expression is increased by cultivation in high glucose-containing media and in diabetic mice, and indicate the involvement of BMPER in high glucose-increased ALP activity in HCASMCs.

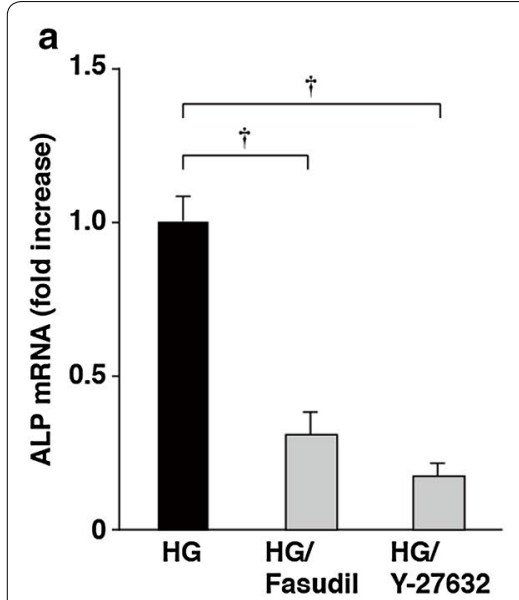

b
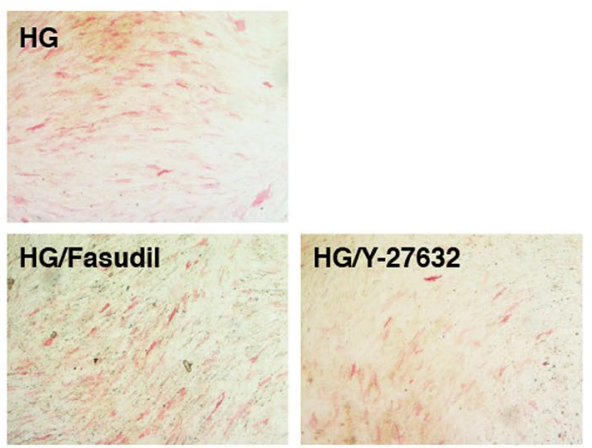

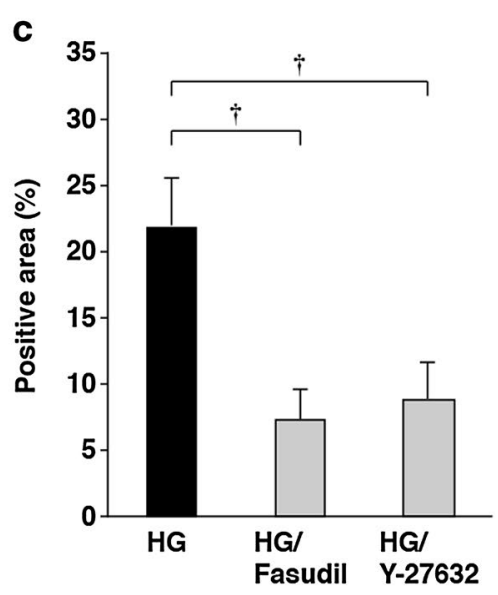

Fig. 2 Inhibition of high glucose-increased ALP mRNA expression and activity by ROCK inhibitors. a Inhibition of high glucose-induced increases in ALP mRNA levels in HCSMCs by fasudil and Y-27632. HCASMCs were cultured in high glucose-containing media for 5 days. The values represent the mean \pm SEM $(n=3)$. b, $\mathbf{c}$ Inhibition of high glucose-induced increases in ALP activity of HCSMCs by fasudil and Y-27632. HCASMCs were cultured for 10 days. Representative images of the ALP-staining (b) and the percentages of the ALP-positive area relative to the total surface area of HCASMCs (c) are shown. The data are presented as mean $\pm \operatorname{SEM}(n=5) .{ }^{\dagger} P<0.01$.
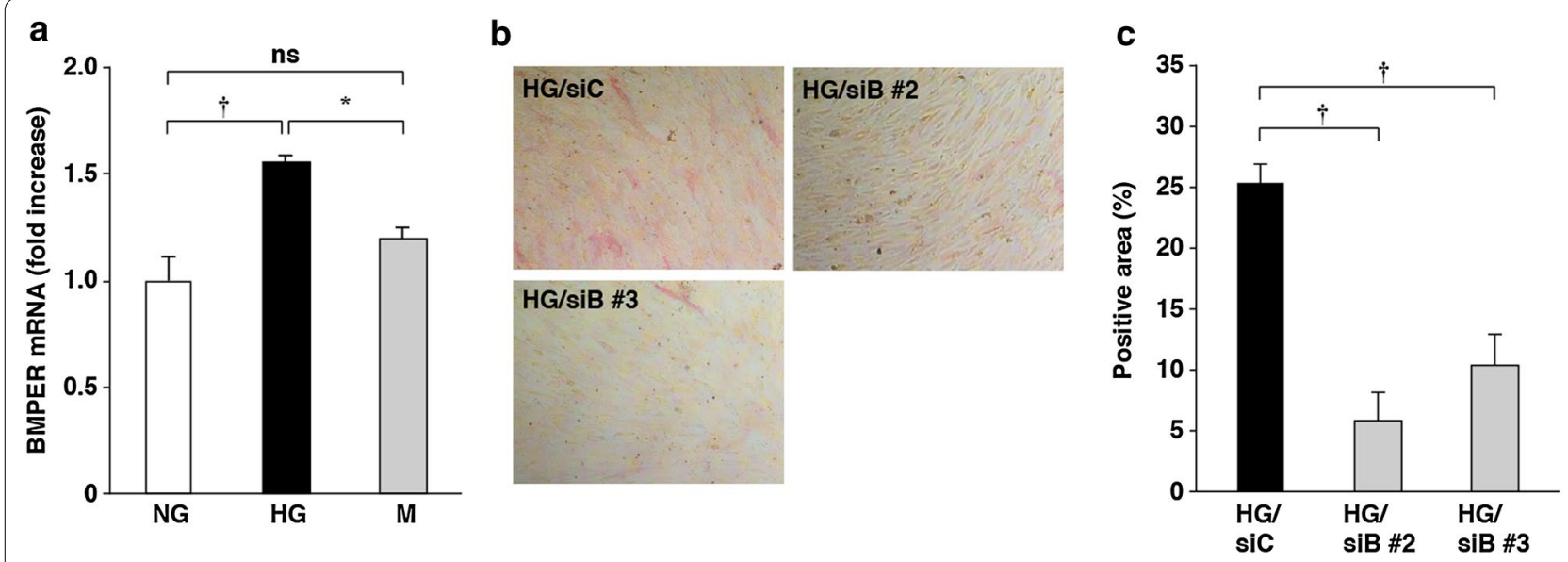

Fig. 3 Involvement of BMPER in high glucose-increased ALP activity. a High glucose-induced increases in BMPER mRNA levels in HCSMCs cultured for 10 days. The values represent the mean \pm SEM $(n=3-4)$. b, c Inhibition of high glucose-induced increases in ALP mRNA levels in HCSMCs by BMPER knockdown. HCASMCs cultured in high glucose-containing media for 10 days. Representative images of the ALP-staining (b) and the percentages of the ALP-positive area relative to the total surface area of HCASMCs $(\mathbf{c})$ are shown. The data are presented as mean \pm SEM $(n=4-5)$. siC control siRNA, siB BMPER siRNA. ${ }^{*} P<0.05 ;{ }^{\dagger} P<0.01$; $n$ s not significant. 


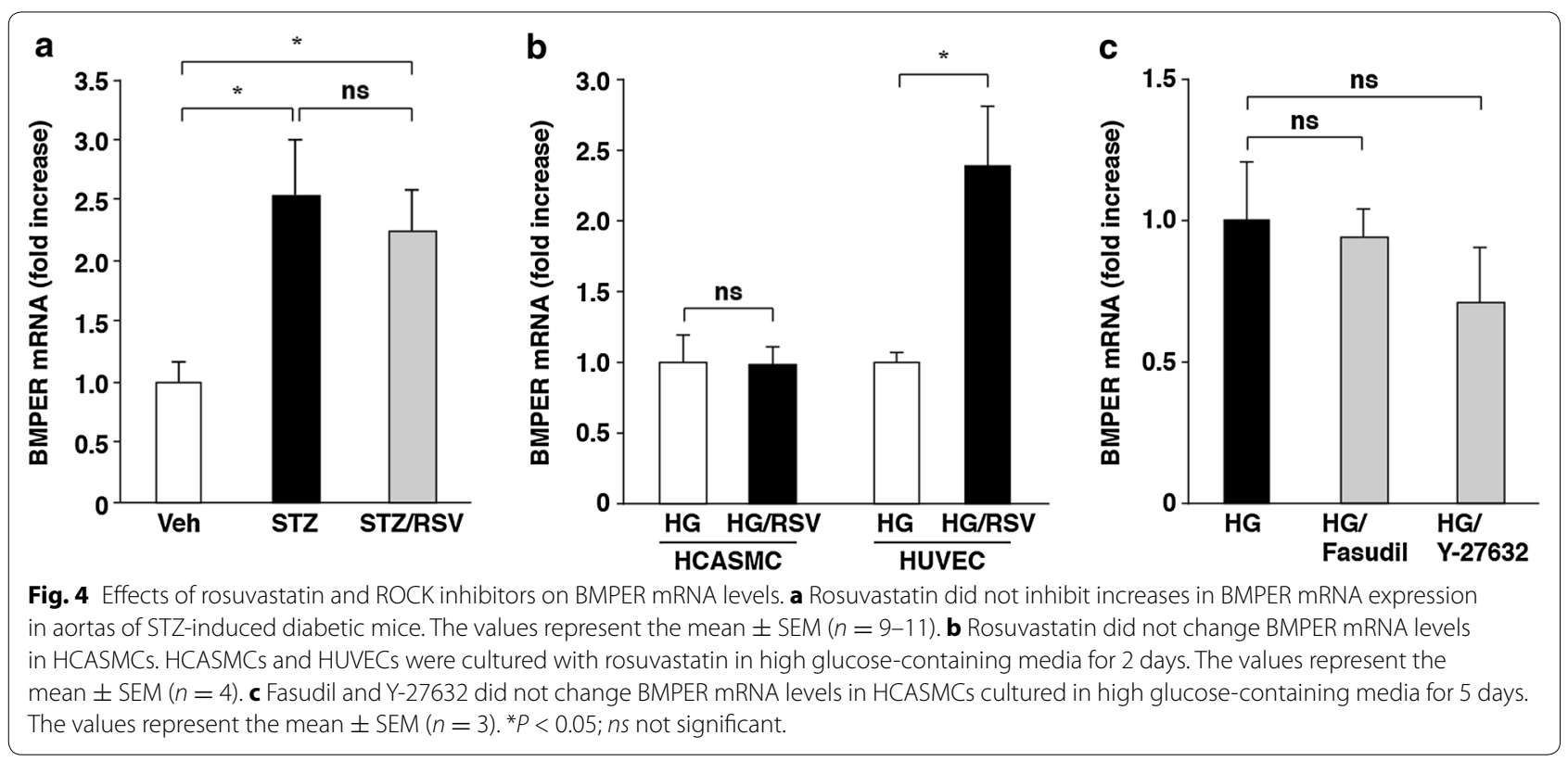

\section{BMPER-mediated ALP activation was independent of the Rho-ROCK signaling pathway}

To clarify the relationship between the Rho-ROCK signaling pathway and BMPER in high glucose-increased ALP activity in HCASMCs, we first examined the effect of rosuvastatin on BMPER mRNA expression. BMPER mRNA expression was not significantly inhibited by rosuvastatin in mouse aortas (Fig. 4a). BMPER mRNA expression was not changed by rosuvastatin in HCASMCs, but was significantly increased in HUVECs (Fig. 4b). The increases in BMPER mRNA expression in HUVECs were consistent with the previous report [24]. Then, the effects of ROCK inhibitors on BMPER mRNA expression were examined. ROCK inhibitors did not inhibit the high glucose-increased BMPER mRNA expression (Fig. 4c). Collectively, these results indicate that the Rho-ROCK signaling pathway is not located upstream of the high glucose-increased BMPER mRNA expression.

Next, to reveal whether high glucose induces activation of the Rho-ROCK signaling pathway via BMPER, we examined MYPT1 phosphorylation. High glucose increased MYPT1 phosphorylation, but knockdown of BMPER did not inhibit MYPT1 phosphorylation (Fig. 5). Collectively, these results indicate that, although the Rho-ROCK signaling pathway is involved in high glucose-induced ALP activation in HCASMCs, BMPERmediated signaling is another pathway independent of the Rho-ROCK signaling pathway.

Finally, the inhibitory effects of BMPER knockdown and rosuvastatin on ALP activity were compared. Although both BMPER knockdown and rosuvastatin showed a significant inhibition of high glucose-increased ALP activity, there still existed a significant inhibitory effect of rosuvastatin (Fig. 6a, b). Collectively, these results suggest that there were at least two pathways in high glucose-increased ALP activity: the Rho-ROCKdependent pathway and the BMPER-dependent pathway.

\section{Discussion}

Critical role of the Rho-ROCK signaling pathway in high glucose-induced ALP activation

Previous reports have shown high glucose induces osteogenic changes in vascular smooth muscle cells [25-27]. Moreover, it has been reported that statins show inhibitory effects on TGF- $\beta$-induced [9], vitamin D3 and warfarin combination therapy-induced [8], and inorganic phosphate-induced [6] trans-differentiation of cultured vascular smooth muscle cells, which is considered to reflect vascular calcification. However, as far as we know, whether statins inhibit high glucose-induced ALP activation has not been reported. Furthermore, we show here, for the first time, the increases in BMPER mRNA expression in vascular smooth muscle cells cultured in high glucose-containing media, and BMPER involvement in high glucose-induced ALP activation, independent of the Rho-ROCK signaling pathway. GGPP is synthesized from the combination of FPP and isopentenyl pyrophosphate (IPP) and because statins block the pathway upstream of these moieties, GGPP cannot be synthesized by treatment with statins even upon FPP supplementation, as IPP is not available [3]. The restoration of rosuvastatin-inhibited ALP activation in HCASMCs by GGPP, 


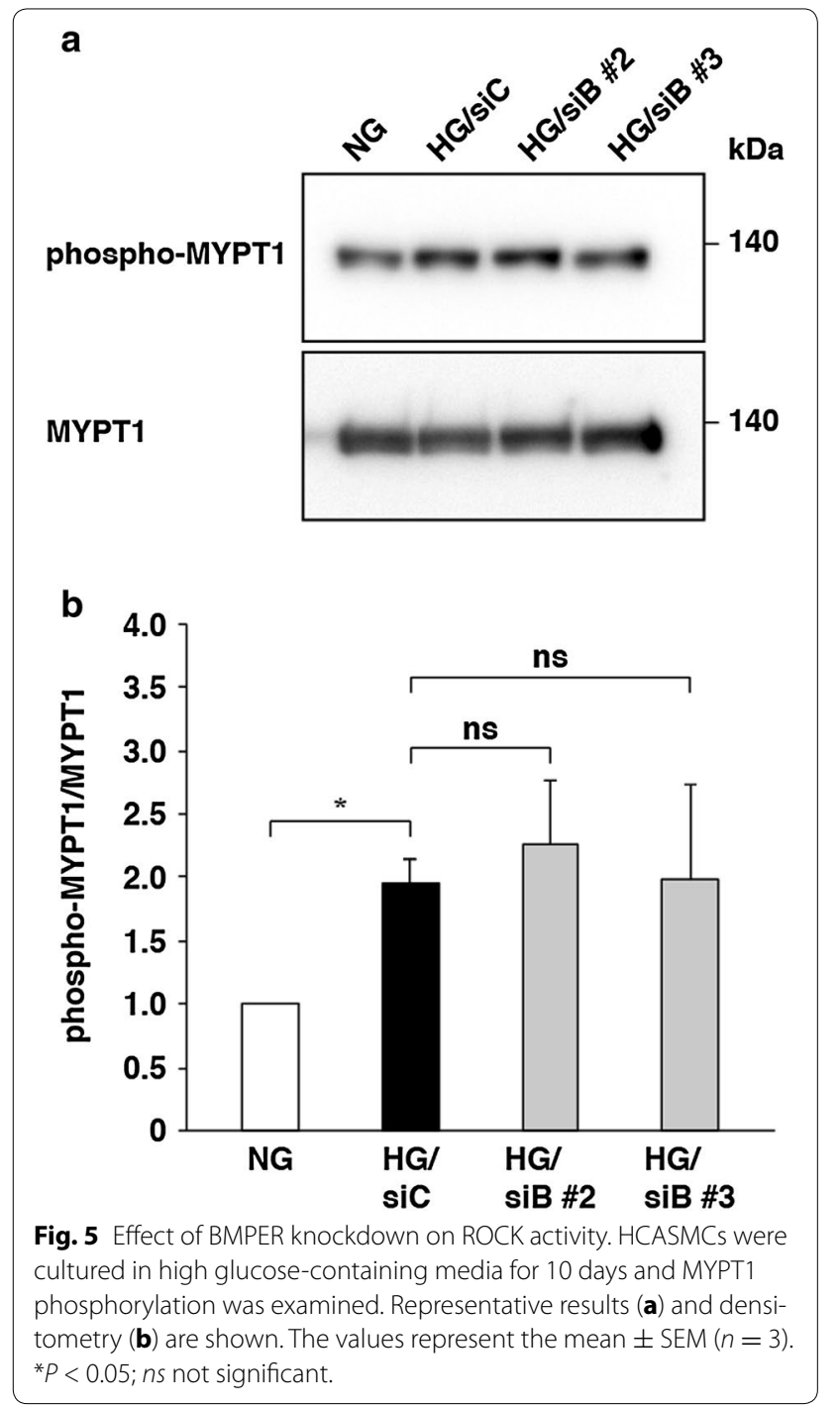

but not by FPP, suggests the involvement of GGPPdependent proteins such as Rho, Rac and Cdc42, rather than farnesylation-dependent proteins such as Ras. In line with this, ROCK inhibitors showed similar inhibitory effects on high glucose-induced ALP activation in HCASMCs. Thus, the Rho-ROCK signaling pathway is critical for high glucose-induced ALP activation.

In contrast to the results of the present study, Trion et al. showed a dose-dependent stimulatory effect of statins on calcification of vascular smooth muscle cells [28]. However, because in this study a 500 times higher concentration of atorvastatin was used than in Son et al. [6], who reported a protective effect of atorvastatin, calcification induced by the statin in the study done by Trion et al. may have resulted from apoptosis of vascular smooth muscle cells. In contrast to lipophilic statins like atorvastatin, hydrophilic statins such as pravastatin and

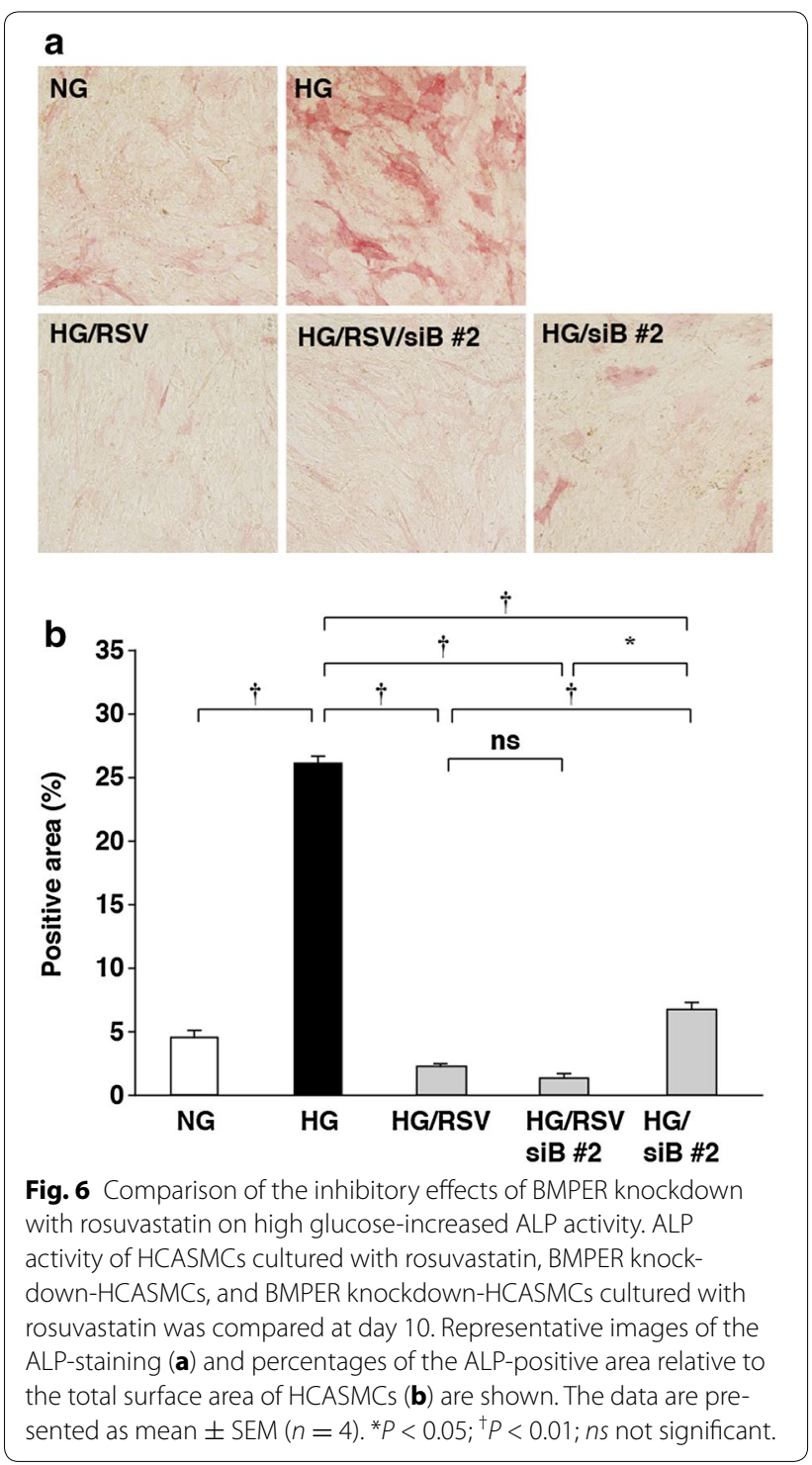

rosuvastatin are reported to suppress apoptosis of various cells [29]. Pravastatin suppresses apoptosis of vascular smooth muscle cells [30-32], while rosuvastatin suppresses apoptosis of vascular endothelial cells [33], cardiomyocytes [34], and podocytes [35]. In the present study, we used rosuvastatin at the commonly-used concentration $(10 \mu \mathrm{M})$, at which apoptosis does not occur, and therefore the rosuvastatin-induced suppression of the high glucose-increased ALP mRNA expression and activity was not due to apoptosis of HCASMCs.

\section{Contribution of the Rho-ROCK signaling pathway and BMPER to high glucose-induced ALP activation}

Tonic ROCK mediated vasoconstriction contributes to coronary vasomotor tone in early diabetes [36], and diabetes-induced vascular dysfunction can arise via 
inhibition of endothelial nitric oxide synthase caused directly or indirectly due to an up-regulation of ROCK by hyperglycemia [37]. Moreover, ROCK is substantially involved in the pathogenesis of coronary vasospasm, angina pectoris, hypertension, pulmonary hypertension, and heart failure [38]. Kawamura et al. have reported that high glucose-increased osteopontin expression was mediated via the Rho-ROCK signaling pathway in vascular smooth muscle cells [25]. We hypothesized that BMPER might be regulated by the Rho-ROCK signaling pathway. However, ROCK inhibitors did not change BMPER mRNA expression. Moreover, BMPER knockdown did not change MYPT1 phosphorylation. Collectively, these results indicate that there are at least two independent pathways in high glucose-induced ALP activation: the Rho-ROCK signaling pathway and the BMPER-dependent pathway (Fig. 7). From our results we hypothesize that there may a possible new therapeutic strategy against vascular calcification, in which BMPER is inhibited. In addition, we have shown here that rosuvastatin and ROCK inhibitors did not have any effects on BMPER mRNA expression in HCASMCs. In contrast, it was reported that statins increased BMPER mRNA expression via the Rho-ROCK signaling pathway in HUVECs [24]. These conflicting results suggest that Bmper gene transcription might be differentially regulated between HCASMCs and HUVECs.

\section{Study limitations}

There are two main limitations to the present study. First, ALP is an essential component of matrix vesicles where it increases for the growth of hydroxyapatite crystal [39]. Furthermore, tissue-nonspecific ALP is distributed in arteries, and is considered to be involved in vascular calcification [39]. We therefore used ALP as

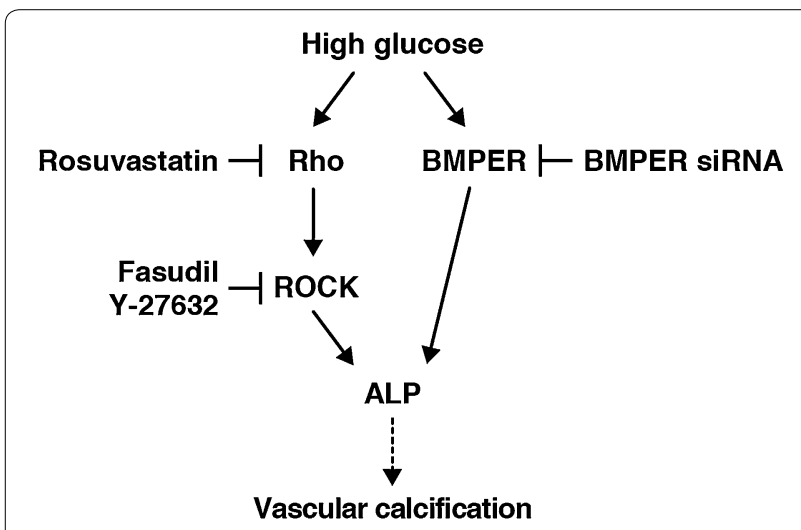

Fig. 7 Contribution of the Rho-ROCK signaling pathway and BMPER to high glucose-induced ALP activation in HCASMCs. There are at least two independent pathways in high glucose-induced ALP activation in HCASMCs: the Rho-ROCK signaling pathway and the BMPER-dependent pathway. surrogate marker of vascular calcification. However, we only examined ALP mRNA levels in vitro and in vivo and ALP activity in vitro, but did not evaluate calcium deposition in vitro and vascular calcification in vivo. It has been shown that calcification does not occur in aortas of STZ-induced type I diabetic rats [40], as is the case with in our experiments. In addition, cultivation in high glucose-containing media does not show calcium deposition in vitro [27]. Therefore, further studies using other animal models of vascular calcification are necessary to evaluate the roles of the Rho-ROCK signaling pathway and the BMPER-mediated signaling pathway in vascular calcification under diabetic conditions.

Another limitation is that in clinical studies whether statins can show an inhibitory effect on vascular calcification is controversial. Kovarnik et al. assessed atherosclerotic plaques using virtual histology intravascular ultrasonography and showed that statins changed the plaques from fibrous and fibro-fatty plaques to necrotic plaques with calcification, suggesting that statins might enhance the density of calcification as part of a healing process [41]. The reason why statins did not show efficacy might be due to their lack of inhibitory effect on progressive calcification, generally detected in clinical imaging, and therefore the starting point to treat might be too late. In the present study, rosuvastatin and BMPER knockdown inhibited ALP activation in HCASMCs, which might reflect a very early stage of vascular calcification.

\section{Conclusions}

Targeting BMPER or BMPER-regulated signaling pathways in vascular smooth muscle cells might be a novel therapeutic target for prevention of vascular calcification.

\section{Abbreviations}

ALP: alkaline phosphatase; BMP: bone morphogenetic protein; BMPER: bone morphogenetic protein-binding endothelial cell precursor-derived regulator; FPP: farnesyl pyrophosphate; GAPDH: glyceraldehyde-3-phosphate dehydrogenase; GGPP: geranylgeranyl pyrophosphate; HCASMC: human coronary artery smooth muscle cell; HMG-CoA: hydroxymethylglutaryl-coenzyme A; HUVEC: human umbilical vein endothelial cell; IPP: isopentenyl pyrophosphate; MYPT1: myosin phosphatase target subunit 1; PCR: polymerase chain reaction; ROCK: Rho-associated protein kinase; RSV: rosuvastatin; SEM: standard error of the mean; siRNA: small interfering RNA; siB: BMPER siRNA; siC: control siRNA; STZ: streptozotocin; TGF: transforming growth factor.

\section{Authors' contributions}

YT, SSK performed the experiments. YT, SSK, KH and YR analyzed the data. YR conceived and designed the experiments. YT, YR wrote the paper. All authors read and approved the final manuscript.

\section{Author details}

${ }^{1}$ Division of Cardiovascular Medicine, Department of Internal Medicine, Kobe University Graduate School of Medicine, 7-5-1 Kusunoki-cho, Chuo-ku, Kobe 650-0017, Japan. ${ }^{2}$ Division of Signal Transduction, Department of Biochemistry and Molecular Biology, Kobe University Graduate School of Medicine, 7-5-1 Kusunoki-cho, Chuo-ku, Kobe 650-0017, Japan. 


\section{Acknowledgements}

Rosuvastatin was kindly provided by AstraZeneca.

\section{Funding}

This work was supported by Grants-in-Aid for Scientific Research from Japan Society for the Promotion of Science (to Y. R.), and grants from the Global Center of Excellence (to K. H. and Y. R.), Takeda Science Foundation, the Mochida Memorial Foundation for Medical and Pharmaceutical Research, and from AstraZeneca (to Y. R.).

Received: 3 July 2015 Accepted: 1 August 2015

Published online: 12 August 2015

\section{References}

1. Chen NX, Moe SM (2003) Arterial calcification in diabetes. Curr Diabetes Rep 3(1):28-32

2. Wang CY, Liu PY, Liao JK (2008) Pleiotropic effects of statin therapy: molecular mechanisms and clinical results. Trends Mol Med 14(1):37-44. doi:10.1016/j.molmed.2007.11.004

3. Goldstein JL, Brown MS (1990) Regulation of the mevalonate pathway. Nature 343(6257):425-430. doi:10.1038/343425a0

4. Van Aelst L, D'Souza-Schorey C (1997) Rho GTPases and signaling networks. Genes Dev 11(18):2295-2322

5. Rikitake Y, Liao JK (2005) Rho GTPases, statins, and nitric oxide. Circ Res 97(12):1232-1235. doi:10.1161/01.res.0000196564.18314.23

6. Son BK, Kozaki K, lijima K, Eto M, Kojima T, Ota H et al (2006) Statins protect human aortic smooth muscle cells from inorganic phosphateinduced calcification by restoring Gas6-AxI survival pathway. Circ Res 98(8):1024-1031. doi:10.1161/01.RES.0000218859.90970.8d

7. Kizu A, Shioi A, Jono S, Koyama H, Okuno Y, Nishizawa Y (2004) Statins inhibit in vitro calcification of human vascular smooth muscle cells induced by inflammatory mediators. J Cell Biochem 93(5):1011-1019. doi:10.1002/jcb.20207

8. Li H, Tao HR, Hu T, Fan YH, Zhang RQ, Jia G et al (2010) Atorvastatin reduces calcification in rat arteries and vascular smooth muscle cells. Basic Clin Pharmacol Toxicol 107(4):798-802. doi:10.1111/j.1742-7843.2010.00580.x

9. Liu D, Cui W, Liu B, Hu H, Liu J, Xie R et al (2014) Atorvastatin protects vascular smooth muscle cells from TGF-beta1-stimulated calcification by inducing autophagy via suppression of the beta-catenin pathway. Cell Physiol Biochem 33(1):129-141. doi:10.1159/000356656

10. Callister TQ, Raggi P, Cooil B, Lippolis NJ, Russo DJ (1998) Effect of HMGCoA reductase inhibitors on coronary artery disease as assessed by electron-beam computed tomography. N Engl J Med 339(27):1972-1978. doi:10.1056/nejm199812313392703

11. Budoff MJ, Lane KL, Bakhsheshi H, Mao S, Grassmann BO, Friedman BC et al (2000) Rates of progression of coronary calcium by electron beam tomography. Am J Cardiol 86(1):8-11

12. Achenbach S, Ropers D, Pohle K, Leber A, Thilo C, Knez A et al (2002) Influence of lipid-lowering therapy on the progression of coronary artery calcification: a prospective evaluation. Circulation 106(9):1077-1082

13. Raggi P, Davidson M, Callister TQ, Welty FK, Bachmann GA, Hecht H et al (2005) Aggressive versus moderate lipid-lowering therapy in hypercholesterolemic postmenopausal women: beyond endorsed lipid lowering with ebt scanning (BELLES). Circulation 112(4):563-571. doi:10.1161/ circulationaha.104.512681

14. Arad Y, Spadaro LA, Roth M, Newstein D, Guerci AD (2005) Treatment of asymptomatic adults with elevated coronary calcium scores with atorvastatin, vitamin C, and vitamin E: the St. Francis Heart Study randomized clinical trial. J Am Coll Cardiol 46(1):166-172. doi:10.1016/j. jacc.2005.02.089

15. Houslay ES, Cowell SJ, Prescott RJ, Reid J, Burton J, Northridge DB et al (2006) Progressive coronary calcification despite intensive lipid-lowering treatment: a randomised controlled trial. Heart 92(9):1207-1212. doi:10.1136/hrt.2005.080929

16. Puri R, Nicholls SJ, Shao M, Kataoka Y, Uno K, Kapadia SR et al (2015) Impact of statins on serial coronary calcification during atheroma progression and regression. J Am Coll Cardiol 65(13):1273-1282. doi:10.1016/j.jacc.2015.01.036
17. Moser M, Binder O, Wu Y, Aitsebaomo J, Ren R, Bode C et al (2003) BMPER, a novel endothelial cell precursor-derived protein, antagonizes bone morphogenetic protein signaling and endothelial cell differentiation. Mol Cell Biol 23(16):5664-5679

18. Serpe M, Umulis D, Ralston A, Chen J, Olson DJ, Avanesov A et al (2008) The BMP-binding protein Crossveinless 2 is a short-range, concentrationdependent, biphasic modulator of BMP signaling in Drosophila. Dev Cell 14(6):940-953. doi:10.1016/j.devcel.2008.03.023

19. Satomi-Kobayashi S, Kinugasa M, Kobayashi R, Hatakeyama K, Kurogane Y, Ishida T et al (2012) Osteoblast-like differentiation of cultured human coronary artery smooth muscle cells by bone morphogenetic protein endothelial cell precursor-derived regulator (BMPER). J Biol Chem 287(36):30336-30345. doi:10.1074/jbc.M111.329110

20. Widyantoro B, Emoto N, Nakayama K, Anggrahini DW, Adiarto S, Iwasa $\mathrm{N}$ et al (2010) Endothelial cell-derived endothelin-1 promotes cardiac fibrosis in diabetic hearts through stimulation of endothelial-tomesenchymal transition. Circulation 121(22):2407-2418. doi:10.1161/ circulationaha.110.938217

21. Tawa H, Rikitake Y, Takahashi M, Amano H, Miyata M, Satomi-Kobayashi S et al (2010) Role of afadin in vascular endothelial growth factor- and sphingosine 1-phosphate-induced angiogenesis. Circ Res 106(11):17311742. doi:10.1161/circresaha.110.216747

22. Rikitake Y, Liao JK (2005) Rho-kinase mediates hyperglycemia-induced plasminogen activator inhibitor-1 expression in vascular endothelial cells. Circulation 111(24):3261-3268. doi:10.1161/circulationaha.105.534024

23. Zhou Q, Liao JK (2010) Pleiotropic effects of statins-basic research and clinical perspectives. Circ J 74(5):818-826

24. Helbing T, Rothweiler R, Heinke J, Goetz L, Diehl P, Zirlik A et al (2010) BMPER is upregulated by statins and modulates endothelial inflammation by intercellular adhesion molecule-1. Arterioscler Thromb Vasc Biol 30(3):554-560. doi:10.1161/atvbaha.109.201087

25. Kawamura H, Yokote K, Asaumi S, Kobayashi K, Fujimoto M, Maezawa Y et al (2004) High glucose-induced upregulation of osteopontin is mediated via Rho/Rho kinase pathway in cultured rat aortic smooth muscle cells. Arterioscler Thromb Vasc Biol 24(2):276-281. doi:10.1161/01. ATV.0000112012.33770.2a

26. Chen NX, Duan D, O'Neill KD, Moe SM (2006) High glucose increases the expression of Cbfa1 and BMP-2 and enhances the calcification of vascular smooth muscle cells. Nephrol Dial Transpl 21(12):3435-3442. doi:10.1093/ ndt/gfl429

27. Sinha A, Vyavahare NR (2013) High-glucose levels and elastin degradation products accelerate osteogenesis in vascular smooth muscle cells. Diabetes Vasc Dis Res 10(5):410-419. doi:10.1177/1479164113485101

28. Trion A, Schutte-Bart C, Bax WH, Jukema JW, van der Laarse A (2008) Modulation of calcification of vascular smooth muscle cells in culture by calcium antagonists, statins, and their combination. Mol Cell Biochem 308(1-2):25-33. doi:10.1007/s11010-007-9608-1

29. Katsiki N, Tziomalos K, Chatzizisis Y, Elisaf M, Hatzitolios Al (2010) Effect of $\mathrm{HMG}-\mathrm{CoA}$ reductase inhibitors on vascular cell apoptosis: beneficial or detrimental? Atherosclerosis 211(1):9-14. doi:10.1016/j. atherosclerosis.2009.12.028

30. Blanco-Colio LM, Villa A, Ortego M, Hernandez-Presa MA, Pascual A, Plaza JJ et al (2002) 3-Hydroxy-3-methyl-glutaryl coenzyme A reductase inhibitors, atorvastatin and simvastatin, induce apoptosis of vascular smooth muscle cells by downregulation of $\mathrm{BCl}-2$ expression and Rho A prenylation. Atherosclerosis 161(1):17-26

31. Knapp AC, Huang J, Starling G, Kiener PA (2000) Inhibitors of HMG-CoA reductase sensitize human smooth muscle cells to Fas-ligand and cytokine-induced cell death. Atherosclerosis 152(1):217-227

32. Sindermann JR, Fan L, Weigel KA, Troyer D, Muller JG, Schmidt A et al (2000) Differences in the effects of HMG-CoA reductase inhibitors on proliferation and viability of smooth muscle cells in culture. Atherosclerosis 150(2):331-341

33. Piconi L, Corgnali M, Da Ros R, Assaloni R, Piliego T, Ceriello A (2008) The protective effect of rosuvastatin in human umbilical endothelial cells exposed to constant or intermittent high glucose. J Diabetes Complicat 22(1):38-45. doi:10.1016/j.jdiacomp.2007.03.004

34. Vilahur G, Casani L, Pena E, Duran X, Juan-Babot O, Badimon L (2009) Induction of RISK by HMG-CoA reductase inhibition affords cardioprotection after myocardial infarction. Atherosclerosis 206(1):95-101. doi:10.1016/j.atherosclerosis.2009.02.009 
35. Cormack-Aboud FC, Brinkkoetter PT, Pippin JW, Shankland SJ, Durvasula RV (2009) Rosuvastatin protects against podocyte apoptosis in vitro. Nephrol Dial Transpl 24(2):404-412. doi:10.1093/ndt/gfn528

36. Pearson JT, Jenkins MJ, Edgley AJ, Sonobe T, Joshi M, Waddingham MT et al (2013) Acute Rho-kinase inhibition improves coronary dysfunction in vivo, in the early diabetic microcirculation. Cardiovasc Diabetol 12:111 doi:10.1186/1475-2840-12-111

37. Cicek FA, Kandilci HB, Turan B (2013) Role of ROCK upregulation in endothelial and smooth muscle vascular functions in diabetic rat aorta. Cardiovasc Diabetol 12:51. doi:10.1186/1475-2840-12-51

38. Shimokawa H, Satoh K (2015) 2015 ATVB plenary lecture: translational research on rho-kinase in cardiovascular medicine. Arterioscler Thromb Vasc Biol 35(8):1756-1769. doi:10.1161/atvbaha.115.305353
39. Wallin R, Wajih N, Greenwood GT, Sane DC (2001) Arterial calcification: a review of mechanisms, animal models, and the prospects for therapy. Med Res Rev 21(4):274-301

40. Wei Q, Ren X, Jiang Y, Jin H, Liu N, Li J (2013) Advanced glycation end products accelerate rat vascular calcification through RAGE/oxidative stress. BMC Cardiovasc Disord 13:13. doi:10.1186/1471-2261-13-13

41. Kovarnik T, Mintz GS, Skalicka H, Kral A, Horak J, Skulec R et al (2012) Virtual histology evaluation of atherosclerosis regression during atorvastatin and ezetimibe administration: HEAVEN study. Circ J 76(1):176-183
Submit your next manuscript to BioMed Central and take full advantage of:

- Convenient online submission

- Thorough peer review

- No space constraints or color figure charges

- Immediate publication on acceptance

- Inclusion in PubMed, CAS, Scopus and Google Scholar

- Research which is freely available for redistribution

Submit your manuscript at www.biomedcentral.com/submit 